\title{
Geometrical and Graphical Representations Analysis of Lissajous Figures in Rotor Dynamic System
}

\author{
Hisham A. H. AL-KHAZALI ${ }^{1} *$, Mohamad R. ASKARI ${ }^{2}$ \\ ${ }^{1}$ Faculty of Science, Engineering and Computing, \\ Kingston University London, School of Mechanical \& Automotive Engineering, \\ London, SW15 3DW, UK \\ ${ }^{2}$ Faculty of Science, Engineering and Computing, \\ Kingston University London, School of Aerospace \& Aircraft Engineering, \\ London, SW15 3DW, UK
}

\begin{abstract}
This paper provides a broad review of the state of the art in Lissajous curve techniques, with particular regard to rotating machinery. Lissajous figure is a subject too wide-ranging to allow a comprehensive coverage of all of the areas associated with this field to be undertaken, and it is not the authors' intention to do so. However, a general overview of the broader issues of Lissajous curve is provided, it tells us about the phase difference between the two signals and the ratio of their frequencies, and several of the various methodologies are discussed.

The experimental technique used Oscilloscopic with Rotor Rig; the display is usually a CRT or LCD panel which is laid out with both horizontal and vertical reference lines we can demonstrate the waveform images on an oscilloscope. The objective of this paper is to provide the reader with an insight into recent developments in the field of Lissajous curve, with particular regard to rotating machines. The subject of it in rotating machinery is vast, including the diagnosis of items such as rotating shafts, gears and pumps.
\end{abstract}

Keywords: Lissajous figure, Oscilloscopic technique, Rotor rig.

\section{INTRODUCTION}

Lissajous figures also called Bowditch curve, pattern produced by the intersection of two sinusoidal curves the axes of which are at right angles to each other. First studied by the American mathematician Nathaniel Bowditch in 1815, the curves were investigated independently by the French mathematician Jules-Antoine Lissajous in 1857-58. Lissajous used a narrow stream of sand pouring from the base of a compound pendulum to produce the curves $[1,2]$.

When using an oscilloscope, we can plot one sinusoidal signal along the $\mathrm{x}$-axis against another sinusoidal signal along the y-axis, the result is a Lissajous figure.

The oscilloscope displays a two dimensional representation of one or more potential differences. The plot is normally of voltage on the $y$-axis against time on the $\mathrm{x}$ axis, making the oscilloscope useful for displaying periodic signals $[3,4]$.

When $x-y$ mode is turned on, the second channel is displayed along the $\mathrm{x}$-axis rather than the time base. For sine waves, this produces a Lissajous Figure from which it is possible to tell the phase difference between the two signals. Lissajous (pronounced LEE-suh-zhoo) figures were discovered by the French physicist Jules Antoine Lissajous. He would use sounds of different frequencies to vibrate a mirror [5].

A beam of light reflected from the mirror would trace patterns which depended on the frequencies of the sounds. Lissajous' setup was similar to the apparatus which is used today to project laser lightshows $[6,7]$.
Before the days of digital frequency meters and phaselocked loops, Lissajous figures were used to determine the

frequencies of sounds or radio signals. A signal of known frequency was applied to the horizontal axis of an oscilloscope, and the signal to be measured was applied to the vertical axis. The resulting pattern was a function of the ratio of the two frequencies $[8,9]$.

Lissajous figures often appeared as props in science fiction movies made during the 1950's. One of the best examples can be found in the opening sequence of the outer limits TV series. ("Do not attempt to adjust your picture-we are controlling the transmission.") The pattern of criss-cross lines is actually a Lissajous figure[10]. The Lissajous Lab provides you with a virtual oscilloscope which you can use to generate these patterns. (You will control the horizontal. You will control the vertical.) The applet also allows you to apply a signal to modulate the hue of the trace, so you can create colourful designs [11, 12].

\section{EXPERIMENTAL FACILITY APPARATUS} AND

\section{II.1 Experimental set-up}

II.1.1 Rotor Rig:- RK4 Rotor Kit made by Bentley Nevada (the advanced power systems energy services company), could be used to extract the necessary information for diagnostic of rotating machinery, such as turbines and 
compressor. Various type of bearing could be used with this rig (plain bearing). The rig must be developed further to find and test new techniques for the classification of problems in monitoring machinery [13]. The rotor kit measures these phenomena with proximity transducers. You can observe the signals from the transducers by using an oscilloscope.

II.1.2 Proximity sensor:- beside the accelerometers, one of the other devices which could be used to obtain the response of a system is proximity probes are sensors able to detect the presence of nearby objects without any physical contact. It often emits an electromagnetic or electrostatic field, or a beam of electromagnetic radiation (infrared for instance) and looks for changes in the field or return signal. The object being sensed is often referred to as the proximity sensors target. The maximum distance that this sensor can detect is defined "nominal range". Proximity sensors can have a high reliability and functional life because of the absence of mechanical parts and lack of physical contact between sensor and the sensed object [14].

II.1.3 Oscilloscope :- the basic oscilloscope, as shown in the illustration, is typically divided into four sections: the display, vertical controls, horizontal controls and trigger controls[15]. In addition to the screen, most display sections are equipped with three basic controls, a focus knob, an intensity knob and a beam finder button, see picture (1).

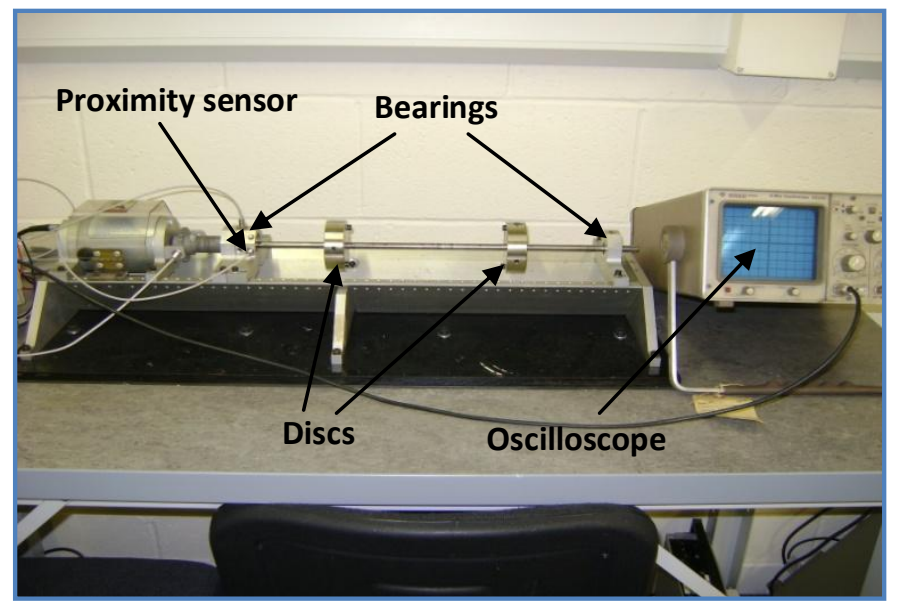

Picture 1: Experimental set-up testing.

\section{II.2 Lissajous figure diagnosis in rotors systems}

Prior to modern computers, Lissajous curves could be generated mechanically by means of a harmonograph [16].

\section{II.2.1 Practical application}

In knot theory, a Lissajous knot is a knot defined by parametric equations of the form

$$
x=\cos \left(n_{x} t+\emptyset_{x}\right), y=\cos \left(n_{y} t+\emptyset_{y}\right), z=\cos \left(n_{z} t+\emptyset_{z}\right)
$$

where $n_{x}, n_{y}$, and $n_{z}$ are integers and the phase shifts $\emptyset_{x}, \emptyset_{y}$, and $\emptyset_{z}$ may be any real numbers.

The projection of a Lissajous knot onto any of the three coordinate planes is a Lissajous curve, and many of the properties of these knots are closely related to properties of it.

In mathematics, a Lissajous curve also known as Lissajous figure, is the graph of a system of parametric equations [17].

$$
x=A \sin (a t+\delta), \quad y=B \sin (b t)
$$

The appearance of the figure is highly sensitive to the ratio $a / b$. For a ratio of 1 , the figure is an ellipse, with special cases including circles $(A=B, \delta=\pi / 2$ radians $)$ and lines $(\delta=0)$. Another simple Lissajous figure is the parabola $(a / b=2, \delta=\pi / 2)$. Other ratios produce more complicated curves, which are closed only if $a / b$ is rational. The visual form of these curves is often suggestive of a threedimensional knot, and indeed many kinds of knots, including those known as Lissajous knots, project to the plane as Lissajous figures.

Lissajous figure on an oscilloscope, displaying a 3:1 relationship between the frequencies of the vertical and horizontal sinusoidal inputs, respectively [2, 12].

Lissajous figures where $a=1, b=N$ ( $N$ is a natural number) and

$$
\delta=\frac{N-1}{N} \frac{\pi}{2}
$$

Lissajous curves can also be generated using an oscilloscope (as illustrated). An octopus circuit can be used to demonstrate the waveform images on an oscilloscope. Two phase-shifted sinusoid inputs are applied to the oscilloscope in $\mathrm{X}-\mathrm{Y}$ mode and the phase relationship between the signals is presented as a Lissajous figure.

On an oscilloscope, we suppose $x$ is $\mathrm{CH} 1$ and $y$ is $\mathrm{CH} 2, A$ is amplitude of $\mathrm{CH} 1$ and $b$ is amplitude of $\mathrm{CH} 2, a$ is frequency of $\mathrm{CH} 1$ and $b$ is frequency of $\mathrm{CH} 2$, so $a / b$ is a 
ratio of frequency of two channels, finally, $\delta$ is the phase shift of $\mathrm{CH} 1$.

A purely mechanical application of a Lissajous curve with $\mathrm{a}=1, \mathrm{~b}=2$ is in the driving mechanism of the Mars light type of oscillating beam lamps popular with railroads in the mid-1900s. The beam in some versions traces out a lopsided Fig. (1), pattern with the "8" lying on its side.

When the input to an LTI system is sinusoidal, the output is sinusoidal with the same frequency, but it may have different amplitude and some phase shift. Using an oscilloscope that can plot one signal against another (as opposed to one signal against time) to plot the output of an LTI system against the input to the LTI system produces an ellipse that is a Lissajous figure for the special case of $a=b$. The aspect ratio of the resulting ellipse is a function of the

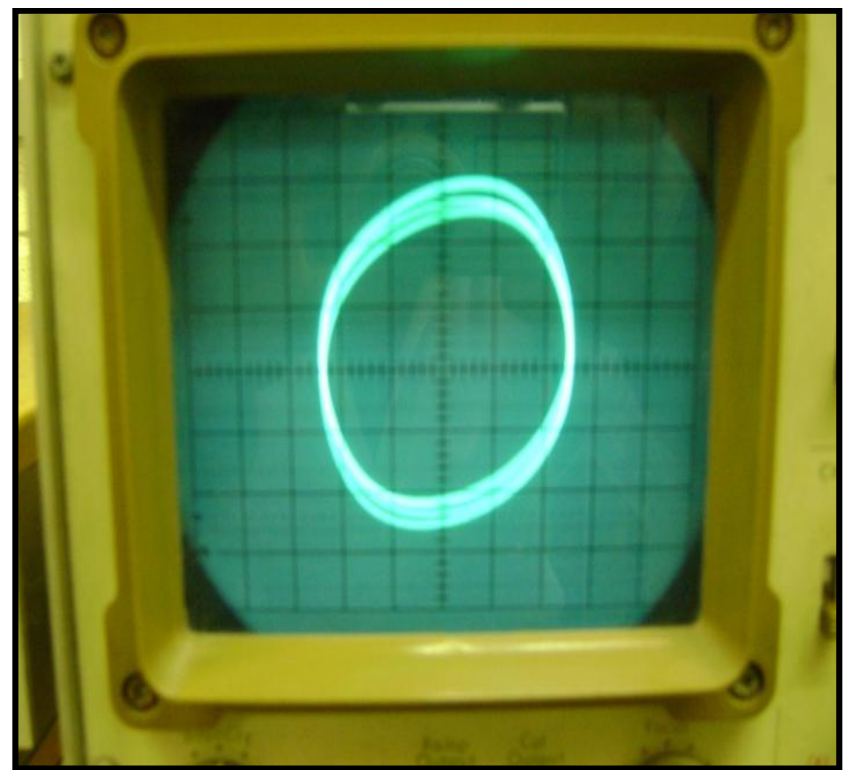

$-\mathrm{A}-$

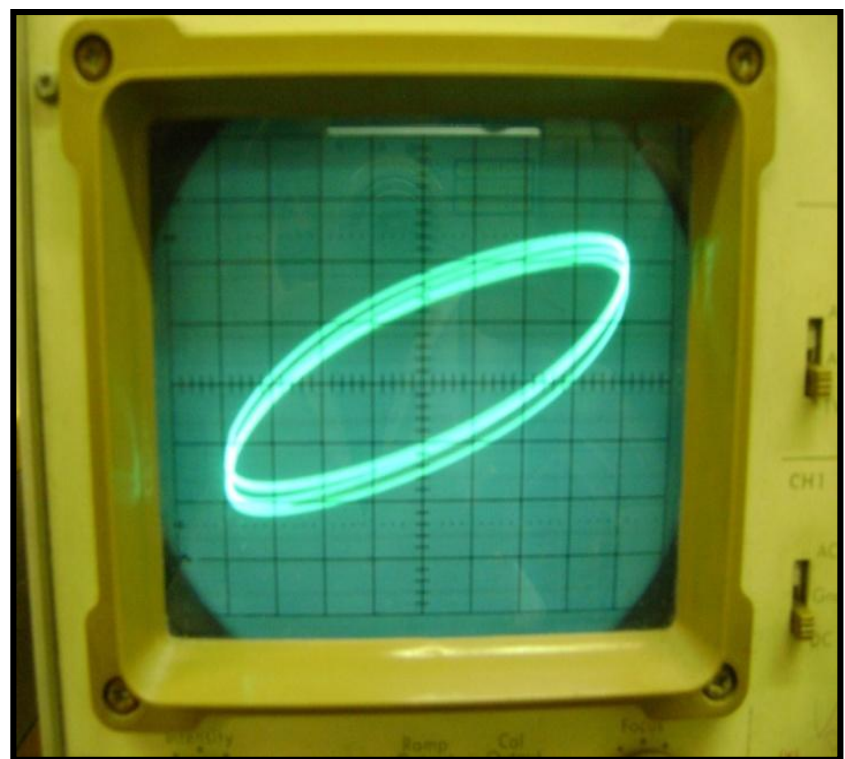

$-\mathrm{C}-$ phase shift between the input and output, with an aspect ratio of 1 (perfect circle) corresponding to a phase shift of $\pm 90^{\circ}$ and an aspect ratio of $\infty$ (a line) corresponding to a phase shift of 0 or $180^{\circ}$ degrees. The picture (2) summarizes how the Lissajous curve changes over different phase shifts. The phase shifts are all negative so that delay semantics can be used with a causal LTI system (note that $-270^{\circ}$ degrees is equivalent to $+90^{\circ}$ degrees). The arrows show the direction of rotation of the Lissajous figure, see Fig.(1-A).

In order to find a resonant frequency you need to search for a Lissajous curve this looks like an ellipse and should be clearly noticeable on the oscilloscope. However it is important to be consistent.

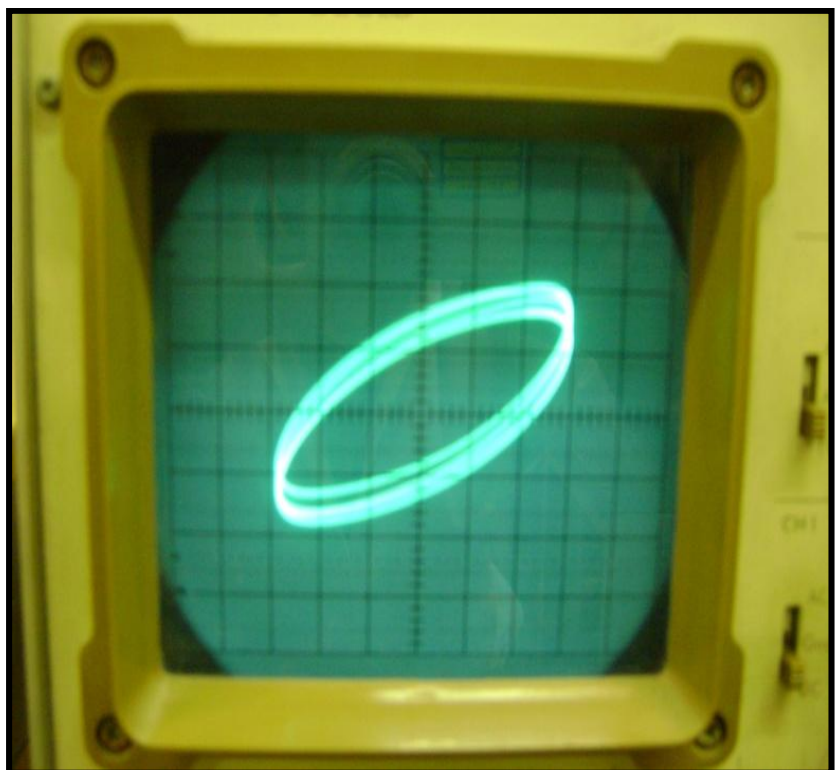

-B-

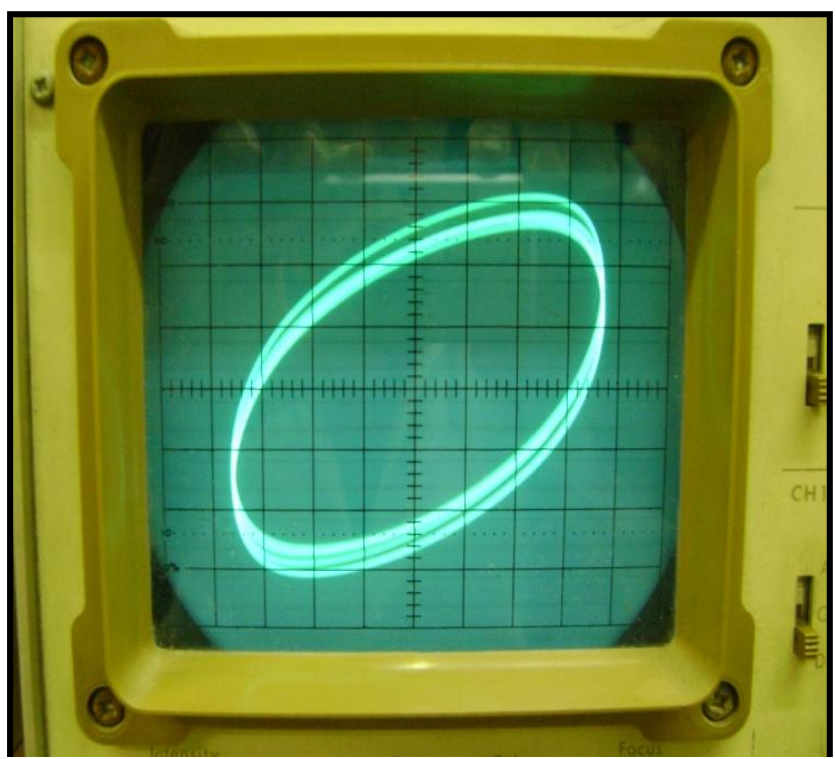

-D- 


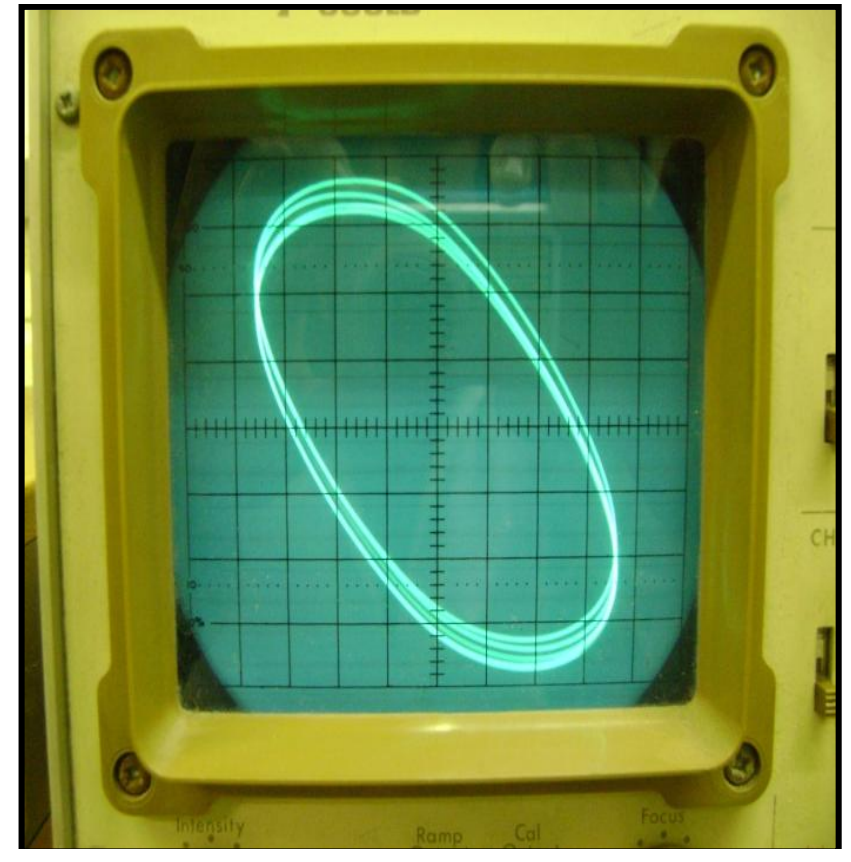

-E-

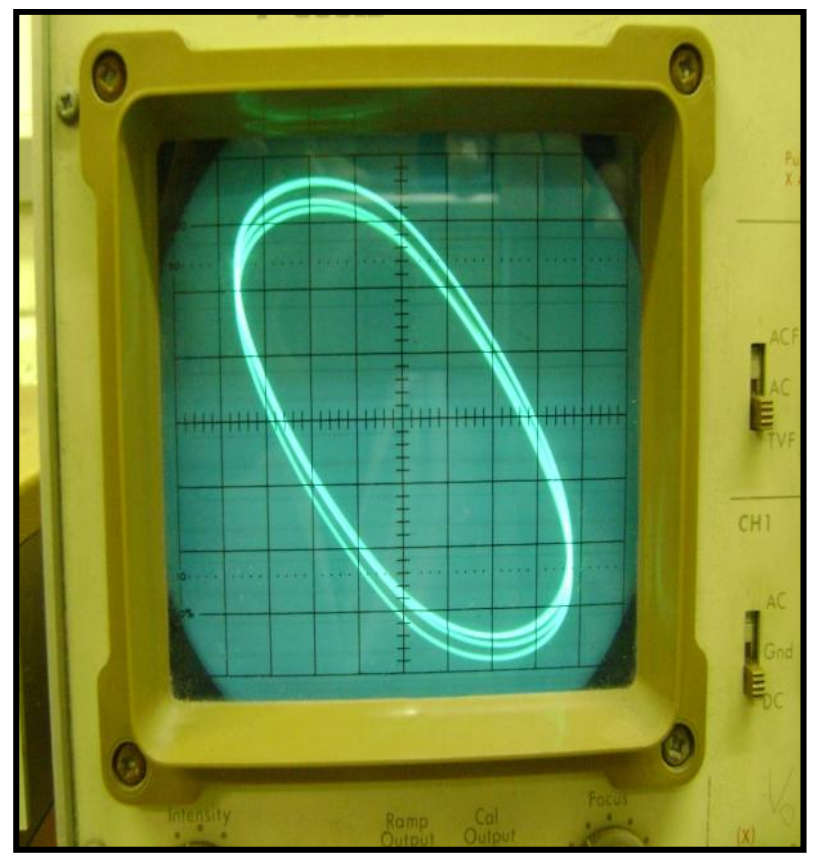

$-\mathrm{F}-$

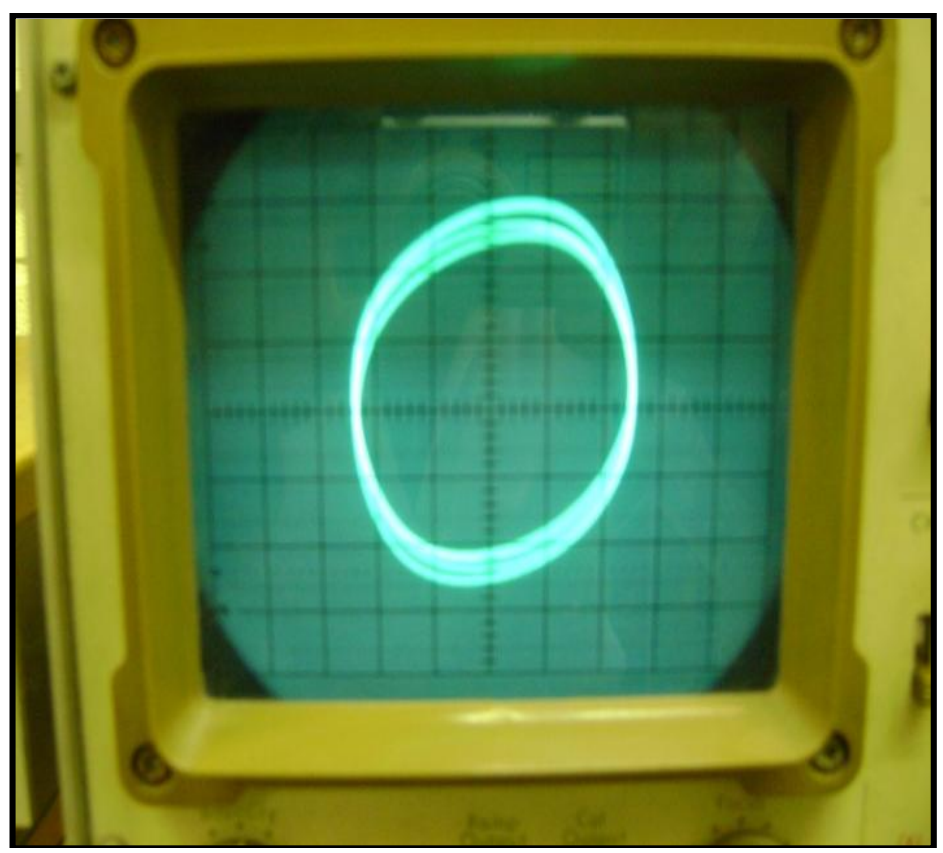

-G-

Picture 2: In this picture both input frequencies are identical, but the phase variance between them creates the shape of an ellipse; 
IOSR Journal of Engineering

May 2012, Vol. 2(5) pp: 971-978

In the following examples the two sine waves have equal amplitudes

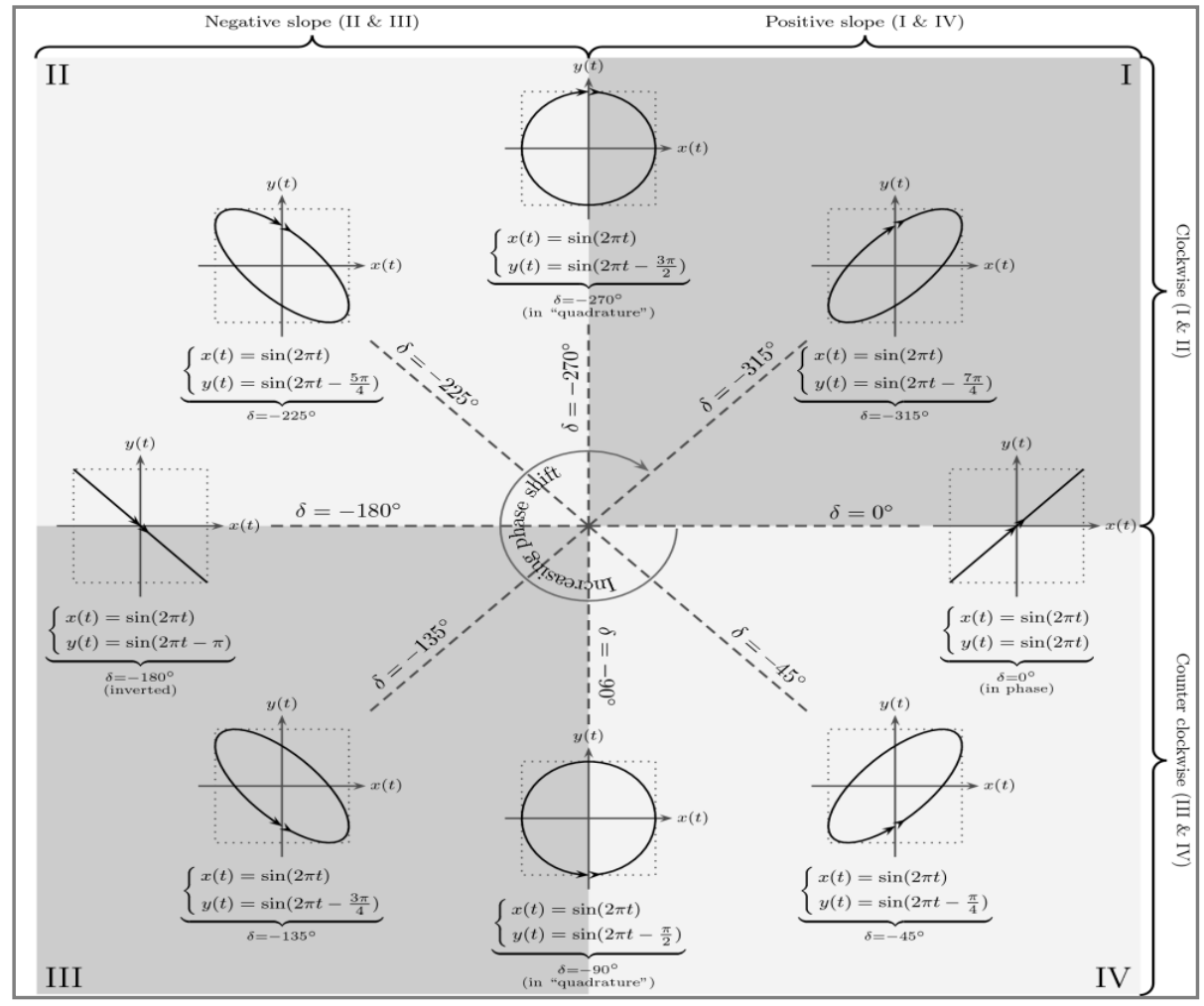

A- For different phase delays.

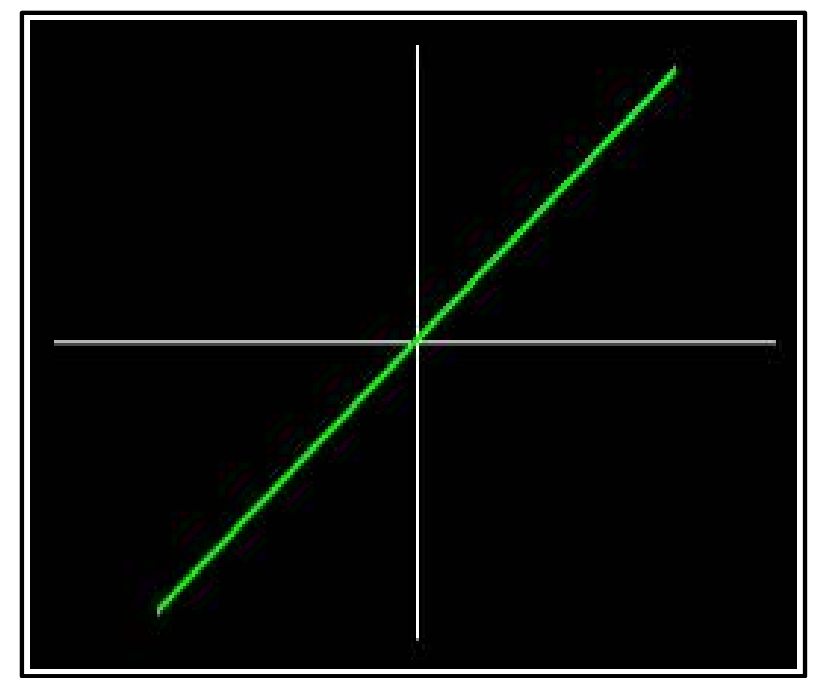

B- When the two sine waves are of equal frequency and in-phase you get a diagonal line to the right. 
IOSR Journal of Engineering

May 2012, Vol. 2(5) pp: 971-978

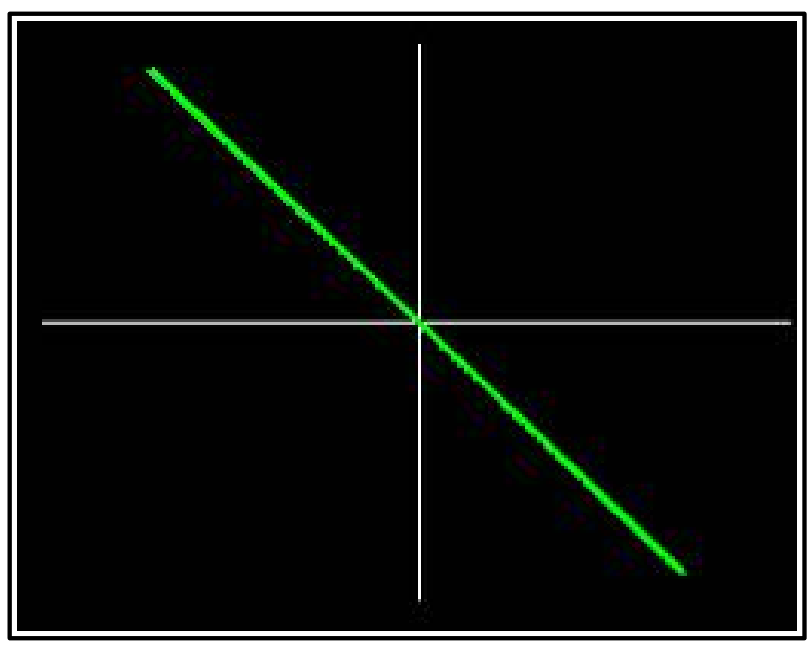

C-When the two sine waves are of equal frequency and $180^{\circ}$ degrees out-of-phase you get a diagonal line to the left.

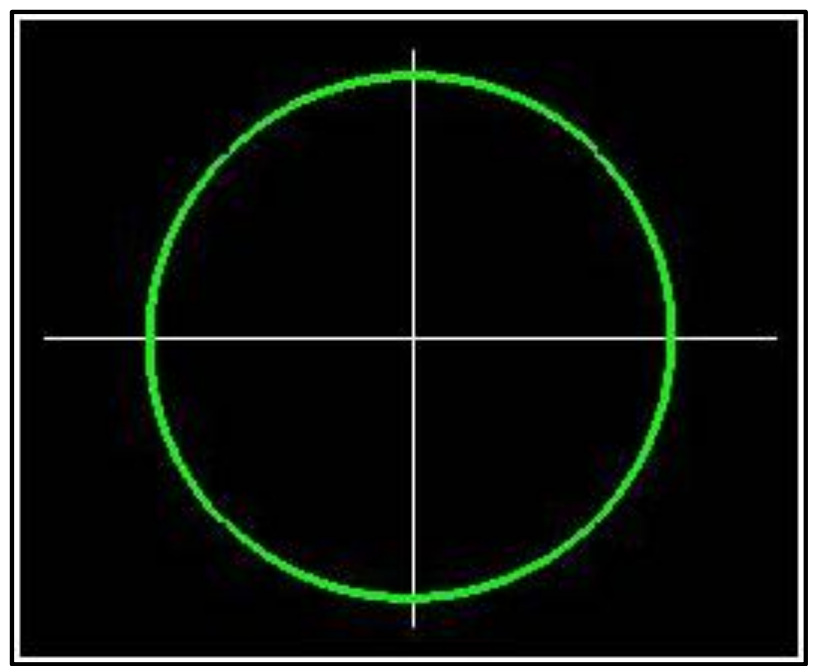

D-When the two sine waves are of equal frequency and $90^{\circ}$ degrees out-of-phase you get a circle.

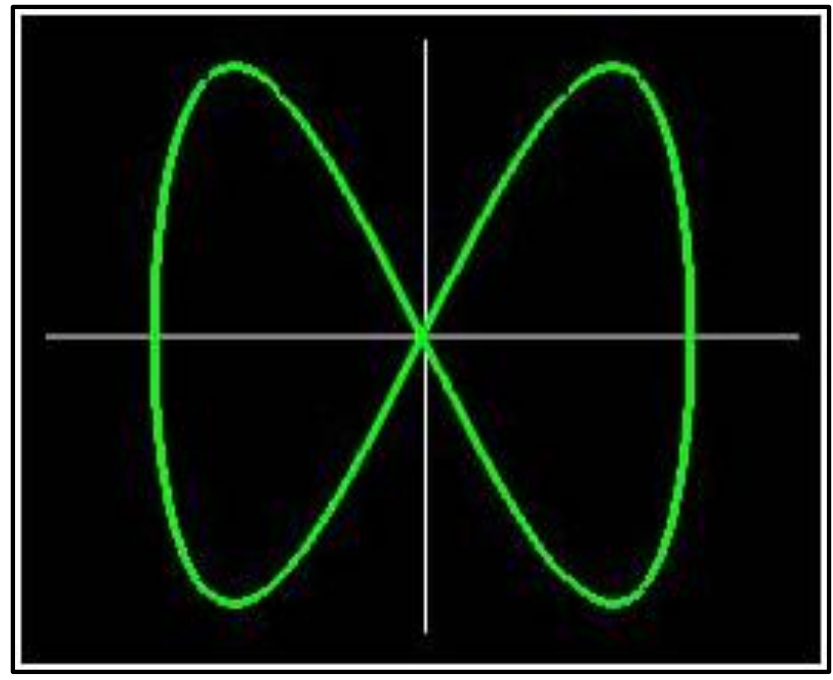

E-Two sine waves, in phase, frequency of horizontal wave twice frequency of vertical wave. 


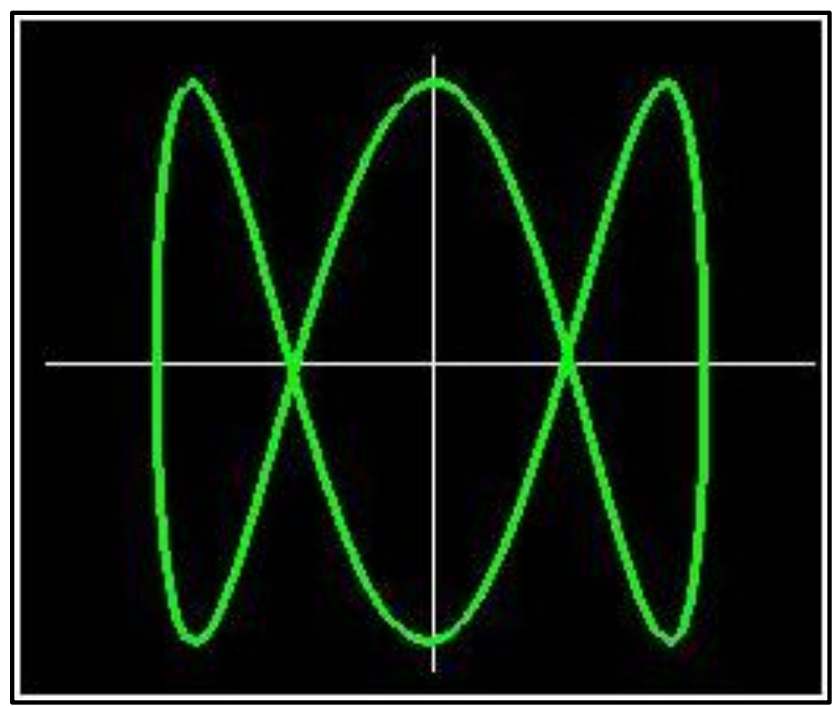

F-Two sine waves, in phase, frequency of horizontal wave three times frequency of vertical wave.

Fig. 1: Showing several Lissajous figures;

This should not be a big surprise, because when

$$
\begin{gathered}
X=\sin (a) \text { and } Y=\sin (a+90)=\cos (a) \\
X^{*} X+Y^{*} Y=\sin (a) * \sin (a)+\cos (a) * \cos (a)=1
\end{gathered}
$$

This is the parametric equation for a circle having a radius of 1 .

\section{CONCLUSION}

A Lissajous figure is produced by taking two sine waves and displaying them at right angles to each other. This is easily done on an oscilloscope in XY mode. It displayed on an oscilloscope can be used to give a quick estimate of the relative phase of two signals at the same frequency. The plots presented here represent

$$
X=\cos (\omega t), \quad Y=\cos (\omega t+\emptyset)
$$

Where the phase angle $\varnothing$ is indicated in degrees by the number the top right of each plot. To use this catalogue, adjust the oscilloscope so that the $X$ and $Y$ signals have exactly the same amplitude ( 8 division's peak-to-peak) the pattern is accurately centred on the screen.

An oscilloscope is easily the most useful instrument available for testing circuits because it allows you to see the signals at different points in the circuit. The best way of investigating an electronic system is to monitor signals at the input and output of each system block

\section{ACKNOWLEDGEMENTS}

The authors are deeply appreciative to (SEC) Faculty of Science, Engineering and Computing in Kingston University London that provides technical support for the research, and the Iraqi Ministry of Higher Education, Iraqi Cultural Attaché in London for funding this work research.

\section{REFERENCES}

[1] H. Cundy, and A. Rollett, Lissajous's figures in mathematical models (3rd ed. Stradbroke, England: Tarquin Pub., 1989).

[2] A. Gray, Modern differential geometry of curves and surfaces with mathematica (2nd ed. Boca Raton, FL: CRC Press, 1997)

[3] J. D. Lawrence, A catalog of special plane curves (New York: Dover, 1972).

[4] Mac Tutor History of Mathematics Archive. Lissajous Curves. http://www-groups.des.stand.ac.uk/ history/Curves/Lissajous.html.

[5] D. Wells, The penguin dictionary of curious and interesting geometry (London: Penguin, 1991).

[6] W. Eric. Weisstein, Lissajous Curve from MathWorld--A Wolfram Web Resource. http://mathworld.wolfram.com/LissajousCurve.html.

[7] Z. Yu-feng, L. Xiao-hui, LI Di-kang, The vibrating frequency and the curve of energy attenuation obtained by using Laser Lissajous Figure, Journal of Foshan University (Natural Science Edition, Physics School of Physics \& Telecommunication Engineering, South China Normal University, Guangzhou 510006, 3, China, 2011.

[8] L. Jianjun, W. Baihai, W. Guocheng, Contrastive research on algorithm for real-time estimate of phase difference of pair of ultrasonic rays, Journal Machine Tool \& Hydraulics; (Faculty of Electromechanical Engineering, Guangdong University of Technology, Guangzhou 510090, 8, China, 2006.

[9] H. Xian-qun, Z. Qing, Using excel study on the synthesis of harmonic vibration, Journal of Science \& Technology Information, Department of Physics and Electronic Engineering, Hanshan Normal University, Chaozhou Guangdong,521041, 1, China, 2010. 
[10] L. Xiao-yong Lu Pei Huang Xu-chu1 Jing Qun Tang Guang-hui, Virtual Simulation of Lissajous Figure by Computer, Journal of Science \& Technology Information; Key Laboratory of Ecology and Department of Physics, Teachers College, Shihezi University, Shihezi Xinjiang, 832003, China; College of Information Science \& Technology, Shihezi University, Shihezi Xinjiang,832003, 13, China, 2010.

[11] Y. Ji-Xian, Study on the properties of lissajous' figures, Journal of Xihua University (Natural Science Edition); Physics Experiment Centre, Xihua University, Chengdu 610039,6, China, 2008.

[12] W. Jian-bo, H. Shi-hua, Z. Jian-long, L. Wei, Theoretical and experimental research of Lissajous figures of square wave, Journal of Physics Experimentation; Department of Physics, Zhejiang Normal University, Jinhua 32100,4, China, 2008.

[13] D. E. Bentley, C. T. Hatch, and B. Grissom, Fundamentals of rotating machinery diagnostics, (Bentley Pressurized Bearing Press, Minden, Nev., USA, 2002).

[14] D. J. Ewins, Modal testing: theory and practice (John wily \& Sons, London, England, 1984).

[15] M.S. Wu and W.H. Tsai, Corrections for Lissajous figures in books, American Journal of Physics, 52, 1984, 657-658.

[16] Y. Ji-xian, Study on the synthesized path of two simple harmonic vibrations with one vertical to another, Journal of Xihua University (Natural Science Edition); (Physics Experiment Centre, Xihua University, Chengdu 610039, 2, China, 2008.

[17] H. Guang, Yiyang, Demonstrating Lissajous figures with MATLAB and their applications, Journal of China Science and Technology Information; Electronic \& Industrial College, Yiyang Hunan 413054, 2, China, 2008.

\section{AUTHORS' INFORMATION}

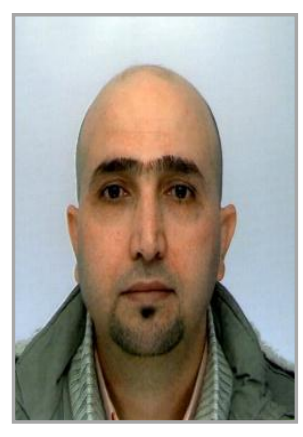

Mr. Hisham A. H. Al-Khazali ${ }^{1}$, $\mathrm{He}$ has $\mathrm{PhD}$ Student in Kingston University London, and (SEM) member, Society for Experimental Mechanics. Inc., in USA. He was born in 28 Aug 1973 Baghdad/Iraq. He received his BEng in Mechanical Engineering (1996), University of Technology, Baghdad. MSc in Applied Mechanics, University of Technology, Baghdad (2000).

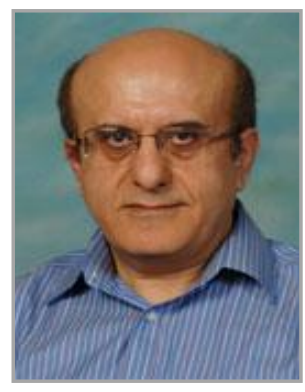

Dr. Mohamad R. Askari ${ }^{2}$ BSc (Eng), MSc, PhD, CEng, MIMechE, MRAeS. He has (Principal Lecturer, Blended Learning Coordinator), Member teaching staff in Kingston University London, His Teaching Area: Applied Mechanics, Aerospace Dynamics, Dynamics and Control, Structural and Flight Dynamics, Engineering Design, Software Engineering to BEng Mechanical and Aerospace second and final years. He was Year Tutor for BEng Mechanical Engineering Course and School Safety Advisor. 